\title{
Internet of Digital Reality: Infrastructural Background - Part II
}

\section{György Wersényi ${ }^{1}$, Ádám Csapó ${ }^{2}$, Tamás Budai ${ }^{1}$, Péter Baranyi ${ }^{2}$}

1 Dept. of Telecommunications, Széchenyi István University

Egyetem tér 1, Győr, Hungary

wersenyi@sze.hu, budai.tamas@sze.hu

2 Dept. of Computer Science, Széchenyi István University

Egyetem tér 1, Győr, Hungary

csapo.adam@sze.hu, baranyi.peter@sze.hu

Abstract: Internet of Digital Reality (IoD) is a concept that extends the Internet of Things (IoT) with the management, transmission and harmonization of digital realities. IoD covers aspects of connectivity, accessibility and usability with respect to different cognitive entities present in the digital world, via a confluence of technologies including virtual reality, artificial intelligence and $2 D$ digital environments, in a way that recognizes human factors and cognitive aspects as key issues. Devices, interfaces, and interacting entities can be enabled through $I o D$ to share digital realities and to thereby build a new level of reality, using intelligent connections mostly based on immersive virtual scenarios and multi-modal interactions in both public and private networks. In this paper, we look into the infrastructural requirements of and challenges behind the Internet of Digital Reality, which must be solved in order to deliver a high-quality user experience while keeping the increasing complexity of these networks at bay.

Keywords: Digital Reality; Future Internet; Internet of Things; Internet of Everything; Internet of Digital Reality

\section{Introduction}

The term 'digital reality' was discussed by the authors in Part I of this paper [1]. There, digital reality was defined as "a high-level integration of virtual reality (including augmented reality, virtual and digital simulations and twins), artificial intelligence and $2 D$ digital environments which creates a highly contextual reality for humans in which previously disparate realms of human experience are brought together".

Internet of Digital Reality (IoD) is a set of technologies that enables digital realities to be managed, transmitted and harmonized in networked environments (both 
public and private), focusing on a higher level of user accessibility, immersiveness and experience with the help of virtual reality and artificial intelligence. Connections among various cognitive entities also have to be handled not only at the end user level of virtual reality displays and software, but also at the levels of network protocols and network management, physical media (wired or wireless), hardware interfaces, and other equipment. AI is a key component of both digital reality and $\mathrm{IoD}$, that enables a cohesion of context-driven content and intelligent network routing to emerge.

Internet of Things (IoT) introduced the world of networked "things" - e.g. sensors and actuators, wearables, digital twins - by integrating distributed computation with intelligent connections. Digital representations of physical entities in the real world can thus be connected, interacted with, managed, and they are able to communicate with each other even without constant human supervision. However, as artificial Intelligence, algorithms, and software robots are becoming sophisticated, reaching levels of partial or full autonomy, making decisions, and being able to evolve and mimic human actors in the virtual world, new possibilities are emerging, based on which the borders between users, operators and digital entities without physical counterparts are becoming blurred. These trends in intelligent data processing, curation and communication, together with the enhanced visual (and increasingly multi-sensory) experience provided by virtual reality technologies (including VR, AR and MR solutions, as detailed in Part I), IoT is gradually evolving into IoD [1]. This in turn raises aspects relevant to network infrastructure and capabilities, as well as various cognitive / human factors aspects, as well as legal and business issues, as described later in this paper.

To better understand the place in history and significance of IoD, it is important to consider how the means of interaction based on which humans can access information has evolved. In the earliest days of commercial IT, up until the late 1980s, the dominant means of interaction with digital systems happened through commandline interfaces (evolved from line terminals), like MS DOS. Then, in the 1990s, 2D graphical user interfaces became widespread (e.g., MS Windows) and the rapid adoption of 2D graphical web pages led to the dotcom boom. A natural next step of this evolution today seems to be to move forward and create 3D operating systems and the 3D Web - in fact, several recent papers clearly show that interactions, communication and analytics in 3D can be considerably more effective than in 2D. This transition, which we may refer to as the "DOS - Windows - Spaces transition" will require us to extend our view and understanding of the key terminologies that are in circulation today ${ }^{1}$. Adopting such a broader perspective is all the more important when we consider how artificial intelligence (AI) and the Internet-based network of AI systems are increasingly becoming a defining feature of our everyday digital environment. In order to increase the effectiveness of AI systems, it is important to enhance the level of communication between humans and AI - after all, data is

$1 \quad$ It should be noted here that although Windows is the name of a proprietary operating system, here we use the word in its most general sense, to refer to the common experience of accessing applications through a $2 \mathrm{D}$ windowing system 
often referred to as "the new oil" [2], without which even AI is powerless. Thus, it is suggested that today's Internet-based economy could benefit from merging with a new kind of 3D digital world enmeshed in big data, digital twins and AI.

In this paper, it is argued that besides changes in the relationship between natural cognitive systems (i.e. humans) and digital devices, a newly emerging, pervasive network of digital cognitive representations is also being formed, which is grounded on a combination of physical, biological and even virtual realities, but also supersedes them in important ways. This network is poised to become so deeply ingrained into daily life that it can be expected to radically alter the basic human experience, and can therefore be referred to as the Internet of Digital Reality. Further, in addition to this general notion of reality being altered, 'digital reality' in this paper is also used in a particular sense of multi-modally accessible integration of VR, AI and 2D digital environments.

\section{Evolution from IoT to IoE}

Internet of Things (IoT) describes the network of physical objects - "things" that are embedded with sensors, software, and other technologies for the purpose of connecting and exchanging data with other devices and systems over the Internet $[3,4,5,6]$.

Through time, the "things" underlying IoT have evolved due to the convergence of multiple technologies, capabilities for real-time analysis / machine learning, commodity sensors, and embedded systems. Traditional fields of embedded systems, wireless sensor networks, control systems, automation (including home and building automation), and others all contribute to enabling the Internet of Things. Today, smartphones and smart "things" each have a cloud representation. Not only for data but year by year more complex AI support. Therefore, whereas Internet of Things (IoT) was one of the first widespread terms used to focus on the connection between "non-human things" from simple sensors to complex highly integrated systems $[7,8,9,10,11,12]$, it is also a concept that is rapidly evolving and changing.

As a result, the concept of IoT has been and continues to be extended / qualified in many different ways. The Internet of Nano Things (IoNT) is a related concept in which the communication exists between nano-scale devices. The Internet of Mission-Critical Things (IoMCT) is used in critical missions such as rescue operations and on battlefields. The Internet of Mobile Things (IoMT) refers to communication between devices using mobile sensors [12].

The Internet of Everything (IoE) was defined by Cisco in 2013 as "bringing together people, process, data, and things to make networked connections more relevant and valuable than ever before, turning information into actions that create new capabilities, richer experiences, and unprecedented economic opportunity for businesses, individuals, and countries" $[13,14]$. In this sense, IoE is the intelligent connection 
of people, processes, data and things. It describes a world where billions of objects have sensors to detect measure and assess their status; all connected over public or private networks using standard and proprietary protocols. There is a fine line of difference between IoE and IoT and that is the intelligent connection. IoT is mostly about physical objects and concepts communicating with each other but IoE is what brings in network intelligence to bind all these concepts into a cohesive system. IoE is an extension of IoT which includes people, process, data and things in network connections.

Pillars of IoE are:

- People: Connecting people in more relevant, valuable ways.

- Data: Converting data into intelligence to make better decisions.

- Process: Delivering the right information to the right entity at the right time.

- Things: Physical devices and objects connected to the Internet and each other for intelligent decision making (IoT) [7]

However, IoE still does not deal with human factors, as the most important part, and other non-technological issues such as business, legal issues, and only partly with safety, security, ergonomics etc. Furthermore, it creates a hard boundary between "people" and "things".

The proliferation of fields called 'Internet-of- $X$ ' (X being something different in each case) somewhat resembles the proliferation of more traditional fields focusing on different kinds of interactions - e.g. human-computer interactions, human-machine interactions, human-robot interactions etc. The emergence of such fields with similar concepts and methodologies (albeit applied to different contexts) was part of the impetus behind the definition of cognitive infocommunications (CogInfoCom) [15, $16,17]$. Similarly, we propose the term 'Internet of Digital Reality (IoD)' to partially integrate, partially complement and more importantly augment earlier notions of the form 'Internet-of-X'. After all, it can be argued that the real motivation behind all of these technological directions was always to merge, augment and share realities - an idea that is already present (excluding the network aspects) in Digital Reality. An overview of the relationship between IoT, IoE and IoD is shown in Figure 1.

\section{Internet of Digital Reality - Technological Challenges}

In one sense, IoD supersedes IoT and IoE where not only physical "things", but also complete digital reality are connected via a (public or private) network.

Today, cognitive entities and virtual / digital twins are strongly based on Internet connections [18]. Pillars of the Internet of Digital Reality include: 


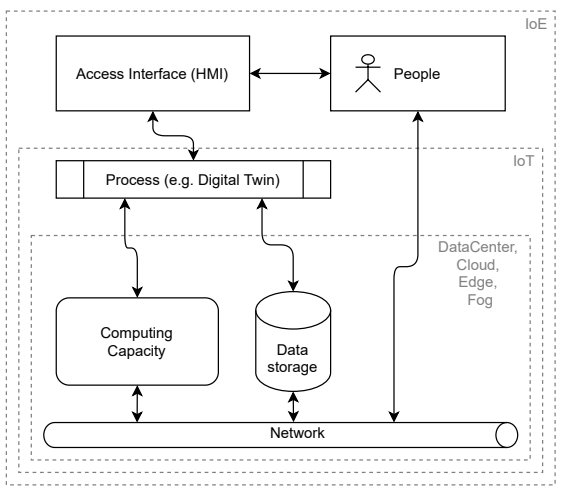

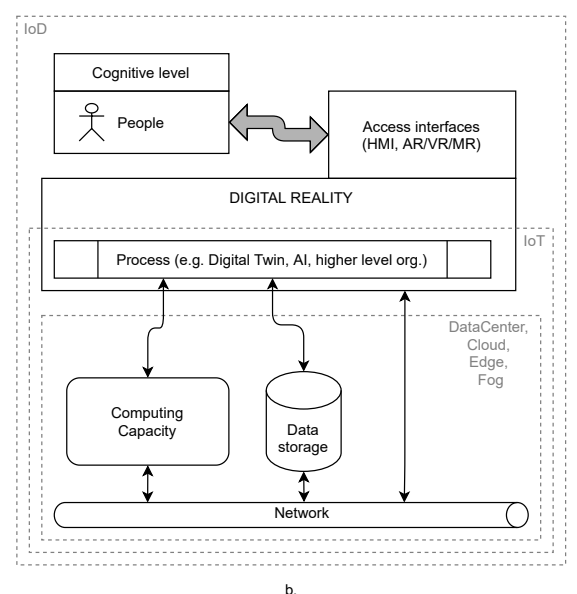

Figure 1

Overview of the different philosophies of IoT, IoE and IoD

- Cognitive entities (such as people, machines, "things", sensors, AI, digital twins, avatars, algorithms, bots, RPA, higher level organizations etc.) interacting in the digital realm.

- Information (data, web content, control).

- Communication networks (intelligent connection, wired and RF physical layers, public and private networks).

- Artificial intelligence, which gains new significance as a global network capable of distributed learning and continued evolution as digital realities become connected and shared across the globe

- Access devices and interfaces (headsets, tactile devices, AR/MR/VR spaces, 360-degree immersive scenarios, mobility and navigation)

- Cognitive infocommunications (sensation and perception, human factors and human-ICT co-evolution)

- Safety and security (data, information, or even the physical safety of the user etc.)

- Digital business and legal issues

- Digital Society (education, acceptance of technology, digital work, e-government, digital arts and gaming)

However, the most relevant aspect of $\mathrm{IoD}$ is that it connects digital realities, that is, combinations of technologies and data that create a higher-level functional integration. For example, IoD is the network through which combinations of $3 \mathrm{D}$ virtual 
spaces and their real-world counterparts can be shared, together with all relevant data and interactive support for extended capabilities.

Since physical things may have digital twins, and in fact any single physical thing can have any number of digital twins, the connections between physical and digital can explode exponentially, causing "Big Data" and intelligent network management to play a crucial role in this equation. As a case in point, the volume and quality of internet traffic requires various improvements and new technological directions. For instance, today a remote cooperation on medical operations requires a very sophisticated and special internet solution in order to overcome latency and safety problems, however, when collaboration on $3 \mathrm{D}$ objects will grow increasingly common in VR environments, this technology will need to be generalised for popular cases, which also necessitates the integration of big data, AI and intelligent internet traffic management including the ability to address various security questions.

\subsection{Cognitive Entities in the Digital World}

Cognitive entities represented digitally are the basis of IoD. Cognitive entities can be conceived of as unique capabilities to effect change that arise via a merging and co-evolution of artificial and natural cognitive systems. This can include human beings, sensors, intelligent algorithms and more. The main characteristic of a cognitive entity is that its capabilities cannot be broken down along the borders between its constituents $[16,19,20]$.

Cognitive entities can interact with each other, can be accessed (some only limited or by permission), and are able for transmit and/or receive information. Furthermore, they are present and "visible" in the virtual reality, thus, they offer access and communication interface of the highest level (e.g. during navigation and movement in the virtual space, via speech or any other interface suitable for communication in the virtual reality).

\subsection{Communication networks}

IoD can be implemented on a variety of communication networks, typically on multi-layered telecommunication networks, but high-speed wired or wireless connection will be a prerequisite. Currently available wired internet networks based on fibre optics, WLAN access points or the growing penetration of 5G (and beyond) mobile internet will serve as a network. Moving toward a future of being online and connected 24/7, RF networks providing access to the internet with mobile devices will stress the importance of $5 \mathrm{G}$ solutions for $\operatorname{IoD}[21,22,9]$.

It is important to emphasize, however, that not only does $\mathrm{IoD}$ depend on specific network technologies, it is also expected to shape them in novel ways, given the new requirements for e.g. QoS, logical network slicing and more, as detailed in Section 4. 


\subsection{Human Factors behind Accessing Devices and Interfaces}

Cognitive infocommunications as a field integrates many aspects of human factors and users' perspective [23, 15, 24, 17]. Cognitive functions create boundaries and limitations around usability and accessibility and system designers have to deal with such issues by adopting a human centered approach.

Interfaces and devices that enable and establish the connections are also important from ergonomic and usability perspective. Especially in the case of headsets (audio and video playback) and VR gloves that give access to a fully tactile experience in IoD, issues of audio/video coding, spatial rendering, 3D vision, multichannel audio playback, sonification, speech production and recognition, spatial resolution of stimuli, latency, ergonomic design, sensation and perception of multimodal excitation signals are just some issues [25, 26, 27, 28].

Human factors, including how human users orientate, navigate, or get access to information influence the accessibility and usability of systems, especially if there are cognitive entities acting like other humans even when they have no human component.

IoD deals not only with the human perspective but also with all the other "needs" for cognitive entities to be operational. Even more importantly, IoD can be expected to shape what is meant by the concept of human factors in a way that is more integrated with the contextual details of daily life - e.g. where a user is situated, what devices a user has access to, what information the user is capable of providing as feedback.

\subsection{Safety and security}

Every communication system, including its components, raises safety issues, and therefore the need for security checks, authentication processes etc. [11]. In the case of IoD this is especially relevant, due to the personal or otherwise sensitive nature of the data involved, and the large variety of different cognitive entities interacting, often without knowing if they are human, artificial, a legal entity or some combination of these. A simple bank transfer that is currently effected on an internetbanking portal of a bank using credit card numbers can be later made in digital reality using gloves or speech communication with a virtual agent. Authentication, verification, transfer of sensitive data is therefore a point behind IoD applications. Furthermore, besides cybersecurity, people's physical safety during operation is a key part in immersive virtual environments (people can fall and get injured, or suffer from simulator sickness etc. [29, 30, 31]). 


\subsection{Digital Business and legal issues}

From a non-technical point of view, a variety of business cases based on Digital Reality (trademarked by Deloitte) have emerged. According to Deloitte's definition, "Digital Reality represents the next digital transformation. It changes how we engage with technology, through augmented-, virtual-, and mixed-reality, 360 video, and immersive experiences that are at once intuitive and data-rich, and which put the human user at the center of design." - with a strong focus on business cases using AR/VR $[32,33,34,35,36,37]$.

A recent analysis of cross-border e-commerce has revealed not only changes in costs related to trading goods but also changes in how people shop, consume, react to online ads and expect goods to be delivered [38]. As IoD is increasingly becoming a centerpiece of such services, new problems related to (and solutions to) various legal aspects are brought to light $[39,40,41]$.

First of all, liability and responsibility in the digital world must be maintained, in order to increase trust in and acceptance of the technology [42, 43]. This aspect is clearly shown e.g. by the case of autonomous driving (i.e. who is to be held responsible when an accident occurs?).

The problem of intellectual property, copyright, original artwork vs. copies is even more significant in the digital and virtual world than before, given the ease with which artifacts can be copied. Virtual money (Bitcoin, Ether and others) and nonfungible tokens (NFT) - all of which are based on blockchain technology (see https://opensea.io/) - are expected to play a key role in this regard. An NFT is a unit of data that certifies a digital asset to be unique and therefore not interchangeable. NFTs can be used to represent the integrity and ownership of various types of digital files. While copies of these digital items are available for anyone to obtain, NFTs are tracked on blockchains to provide the owner with a proof of ownership that is separate from copyright and independent of how many copies of the digital item are made.

In immersive digital reality, where algorithms, deep learning solutions and more broadly speaking artificial intelligence have an active role to play, the question of who has the right to make certain decisions has far-reaching implications. If it is difficult to decide what kind of digital content and cognitive entities are present, and communicating with each other, there is an enormous risk for illegal activity from fraud to international terrorism and other activities.

\subsection{Digital Society}

Acceptance of new technology is a wide area to explore. Society includes not only technically educated individuals and experts, but also children, elderly users and people who are generally not familiar with and wary of new technologies. Increasing users' acceptance levels is a key point in all digital fields, not to mention in IoD 
applications. Use cases in education, work, healthcare, e-government and administrative solutions etc. can help show the positive side of digital realities [44].

A Digital Society is a group of people who can use digital technologies to their benefit. The recent Coronavirus pandemic has shown how important it is for groups of people to be familiar with the same technological possibilities, whether they be related to education, shared working and / or home office environments, or whether they be simple tools of communication (as in the case of Zoom, Teams or Google Meet) $[45,46,35]$. Applications related to the fine arts and performing arts can also help familiarize soceity with the technological possibilities and far-reaching impacts of Digital Reality.

\section{Connecting Digital Realities}

Efficiency in communication is a key factor in any network. Efficiency can be increased if the network management is optimized for the requirements of common use cases and if the network can understand what entities are present and connected in the network. Network design and management optimization can be enhanced if the components of a digital reality can be grouped by some logic and functions in an actual task. Furthermore, they can be re-grouped or be a part of different set(s) of realities [47].

As an example, consider an 'integration' of a physical refrigerator, microwave oven, smart stove and deep fryer along with their corresponding digital twins, together with various further virtual objects in a shareable VR space, which also includes a 2D digital environment of collaborative cookbooks, cooking notes, cooking blogs, and video chats with a connection to the most renowned experts in the culinary arts - an example already detailed in Part I of this paper [1]. In such a scenario, the oven, stove and deep fryer, along with the various sensors, tablet displays etc. share the same physical network and instead of being independent or loosely connected, they may be logically grouped under the "cooking" label. At the same time, the smart fridge can be a member in the "what to buy at the grocery" group and the tablet can stream music as a member of the "infotainment" group. This might fit into the usual smart home and IoT concept, but IoD extends this in order for all of these components to be present in the same, integrated reality such that the user has access to all the relevant information and capabilities through AR/MR/VR glasses and/or haptic/tactile devices, voice commands and speech feedback and even AI-driven interfaces (e.g. chatbots) in a fully immersive way.

If the network is "smart" enough to be able to understand logically grouped components, it can be configured dynamically (and in an autonomous way), providing easy access for the user. The specific level of safety and security, communication and network management capabilities and key performance metrics (data rate, latency etc.) can be tailored to current requirements at any given time. 
Increasing user experience relies also on reducing latency (increasing speed of control and feedback) in the system. By latency here we mean the time needed for handling an event initialized by inner status change of a component in Digital Reality, including the time for accessing, processing and transmitting of data over the network. The latency can be mission critical in some cases (real-time surgery, selfdriving vehicle) in the order of milliseconds, but it can be also in the order of seconds, where time is not a limiting factor of the outcome.

Nowadays, different methods exist in computer networks for decreasing latency. In the case of IoD the following methods can be used or reconsidered, adapted to the needs of the network:

- QoS: different data types are sensitive to latency in different ways. The Quality of Service depends on the type of data (audio, video, etc.). In the scope of IoD QoS could be extended by differentiating network traffic not only by data-stream type, but by taking into account the logical grouping of entities.

- Logical Network Slicing: it would be beneficial, if logically grouped and geographically co-located digital entities shared the same physical network if possible allowing the shortest path for data transfer. Furthermore, fully digital entities such as algorithms, AIs not necessarily are near or of known origin. From the network side, a Software Defined Network can be helpful (that is a first class citizen in 5G), and from teh computing/resource side Cloud, Edge and Fog Computing.

- Multipath routing: in order to ensure low latency and add redundancy different network endpoints (e.g. WLAN, Mobile broadband and wired LAN) can be grouped and used together to transmit a single data stream [48].

- CDN: the idea behind a Content Delivery Network is that a logical digital entity (e.g. a movie file with audio and video) can be copied and multiplied. Copies then will be stored at different locations (servers), and users access the nearest provider for download or streaming. The question of copying is not only a technical problem (how do we make a replica of an AI?), but also a legal problem (property rights, copyright).

\section{Conclusions}

Part I of this paper introduced the concept of Digital Reality, which allows users to access, manipulate and interact with integrated sets of content - whether on display screens, immersive settings or overlaid on physical objects - that represent concepts and / or objects that can be both physical or digital, as well as both real or imagined. In Part II, an overview was given on Internet of Things and Internet of Everything, followed by a detailed discussion on the concept of Internet of Digital Reality. The discussion covered aspects relevant to technology, business use cases, legal issues and societal factors. 
Acknowledgements This research was supported by the Digital Development Center in the national framework GINOP-3.1.1-VEKOP-15- 2016-00001 "Promotion and support of cooperations between educational institutions and ICT enterprises".

\section{References}

[1] P. Baranyi, Á. Csapó, T. Budai, and G. Wersényi, "Introducing the Concept of Internet of Digital Reality - Part I," Acta Polytechnica Hungarica, vol. 18, no. 7, pp. 225-240, 2021.

[2] C. Humby, "Data is the New Oil," Proc. ANA Sr. Marketer's Summit. Evanston, IL, USA, 2006.

[3] M. Rouse, "Internet of Things." https://internetofthingsagenda . techtarget.com/definition/Internet-of-Things-IoT, 2019.

[4] E. Brown, "21 Open Source Projects for IoT." https://www. linux.com/ news/21-open-source-projects-iot/, 2016.

[5] ITU, "Internet of Things Global Standards Initiative," 2015.

[6] P. A. Laplante, M. Kassab, N. L. Laplante, and J. M. Voas, "Building caring healthcare systems in the Internet of Things," IEEE Systems Journal, vol. 12, no. 3, pp. 3030-3037, 2017.

[7] A. Banafa, "The Internet of Everything (IoE)," Aug 2018.

[8] S. Greengard, The Internet of Things. MIT press, 2015.

[9] S. Li, L. Da Xu, and S. Zhao, "5G Internet of Things: A survey," Journal of Industrial Information Integration, vol. 10, pp. 1-9, 2018.

[10] J. H. Nord, A. Koohang, and J. Paliszkiewicz, "The Internet of Things: Review and theoretical framework," Expert Systems with Applications, vol. 133, pp. 97$108,2019$.

[11] F. A. Alaba, M. Othman, I. A. T. Hashem, and F. Alotaibi, "Internet of Things security: A survey," Journal of Network and Computer Applications, vol. 88, pp. 10-28, 2017.

[12] C. Srinivasan, B. Rajesh, P. Saikalyan, K. Premsagar, and E. S. Yadav, "A Review on the Different Types of Internet of Things (IoT)," Journal of Advanced Research in Dynamical and Control Systems, vol. 11, no. 1, pp. 154-158, 2019.

[13] Cisco, "The Internet of Everything - Global Private Sector Economic Analysis." https://www.cisco.com/c/dam/en_us/about/ac79/docs/ innov/IoE_Economy_FAQ.pdf, 2013.

[14] Cisco, "The Internet of Everything - Cisco IoE Value Index Study." https: //www.cisco.com/c/dam/en_us/about/business-insights/ docs/ioe-value-index-faq.pdf, 2013. 
[15] P. Baranyi and Á. Csapó, "Definition and synergies of cognitive infocommunications," Acta Polytechnica Hungarica, vol. 9, no. 1, pp. 67-83, 2012.

[16] P. Baranyi, A. Csapo, and G. Sallai, Cognitive Infocommunications (CogInfoCom). Springer, 2015.

[17] J. Katona, "A Review of Human-Computer Interaction and Virtual Reality Research Fields in Cognitive InfoCommunications," Applied Sciences, vol. 11, no. 6, p. 2646, 2021.

[18] D. Jones, C. Snider, A. Nassehi, J. Yon, and B. Hicks, "Characterising the Digital Twin: A systematic literature review," CIRP Journal of Manufacturing Science and Technology, vol. 29, pp. 36-52, 2020.

[19] P. Baranyi and A. B. Csapo, "Revisiting the concept of generation CEGeneration of Cognitive Entities," in 2015 6th IEEE International Conference on Cognitive Infocommunications (CogInfoCom), pp. 583-586, IEEE, 2015.

[20] L. I. Komlósi and P. Waldbuesser, "The cognitive entity generation: Emergent properties in social cognition," in 2015 6th IEEE International Conference on Cognitive Infocommunications (CogInfoCom), pp. 439-442, IEEE, 2015.

[21] E. Bastug, M. Bennis, M. Médard, and M. Debbah, "Toward interconnected virtual reality: Opportunities, challenges, and enablers," IEEE Communications Magazine, vol. 55, no. 6, pp. 110-117, 2017.

[22] A. A. Shchurov, "A multilayer model of computer networks," arXiv preprint arXiv:1509.00721, 2015.

[23] W. P. Neumann, S. Winkelhaus, E. H. Grosse, and C. H. Glock, "Industry 4.0 and the human factor-A systems framework and analysis methodology for successful development," International Journal of Production Economics, vol. 233, p. 107992, 2021.

[24] Á. Csapó and G. Wersényi, "Overview of auditory representations in humanmachine interfaces," ACM Computing Surveys (CSUR), vol. 46, no. 2, pp. 1-23, 2013.

[25] L. Xue, C. J. Parker, and H. McCormick, "A virtual reality and retailing literature review: Current focus, underlying themes and future directions," Augmented Reality and Virtual Reality, pp. 27-41, 2019.

[26] J. L. Rubio-Tamayo, M. Gertrudix Barrio, and F. García García, "Immersive environments and virtual reality: Systematic review and advances in communication, interaction and simulation," Multimodal Technologies and Interaction, vol. 1, no. 4, p. 21, 2017.

[27] Á. Csapó, G. Wersényi, H. Nagy, and T. Stockman, "A survey of assistive technologies and applications for blind users on mobile platforms: a review 
and foundation for research," Journal on Multimodal User Interfaces, vol. 9, no. 4, pp. 275-286, 2015.

[28] S. Spagnol, G. Wersényi, M. Bujacz, O. Bălan, M. Herrera Martínez, A. Moldoveanu, and R. Unnthorsson, "Current use and future perspectives of spatial audio technologies in electronic travel aids," Wireless Communications and Mobile Computing, vol. 2018, 2018.

[29] E. Chang, H. T. Kim, and B. Yoo, "Virtual reality sickness: a review of causes and measurements," International Journal of Human-Computer Interaction, vol. 36, no. 17, pp. 1658-1682, 2020.

[30] K. Stanney, B. D. Lawson, B. Rokers, M. Dennison, C. Fidopiastis, T. Stoffregen, S. Weech, and J. M. Fulvio, "Identifying Causes of and Solutions for Cybersickness in Immersive Technology: Reformulation of a Research and Development Agenda," International Journal of Human-Computer Interaction, vol. 36, no. 19, pp. 1783-1803, 2020.

[31] S. Nichols and H. Patel, "Health and safety implications of virtual reality: a review of empirical evidence," Applied ergonomics, vol. 33, no. 3, pp. 251-271, 2002.

[32] Deloitte, "The Internet of Everything (IoE)." https://www2.deloitte. $\mathrm{com} /$ us/en/pages/consulting/topics/digital-reality. html, 2021.

[33] D. O. Baecker, "Digital Reality explained... in under 100 words." https://www2.deloitte.com/ch/en/pages/innovation/ articles/digital-reality-explained.html, 2021.

[34] P. D. Ramani Moses, Nikita Garia, "Digital Reality A technical primer." https://www2.deloitte.com/ insights/us/en/topics/emerging-technologies/ digital-reality-technical-primer.html, 2021.

[35] E. Mitrofanova, I. Bogatyreva, and V. Tarasenko, "Digital reality and perspective of the management of educational organizations," in International Scientific Conference "Digital Transformation of the Economy: Challenges, Trends, New Opportunities”, pp. 390-397, Springer, 2019.

[36] S. Sharma and C. Bach, "An exploratory research on virtual reality and how it affects future of shopping and immerging fields," European Journal of Engineering and Technology Research, vol. 1, no. 6, pp. 34-43, 2016.

[37] N. O. Stolyarov, E. S. Petrenko, O. A. Serova, and A. S. Umuralieva, "The digital reality of the modern economy: new actors and new decision-making logic," in Institute of Scientific Communications Conference, pp. 882-888, Springer, 2019. 
[38] E. Gomez-Herrera, B. Martens, and G. Turlea, "The Drivers and Impediments for Cross-Border E-Commerce in the EU," Information Economics and Policy, vol. 28, pp. 83-96, 2014.

[39] M. R. Carrillo, "Artificial intelligence: From ethics to law," Telecommunications Policy, vol. 44, no. 6, p. 101937, 2020.

[40] J. S. Spiegel, "The ethics of virtual reality technology: social hazards and public policy recommendations," Science and engineering ethics, vol. 24, no. 5, pp. 1537-1550, 2018.

[41] W. K. Robinson and J. T. Smith, "Emerging Technologies Challenging Current Legal Paradigms," Minn. JL Sci. \& Tech., vol. 19, p. 355, 2018.

[42] K. Siau and W. Wang, "Building trust in artificial intelligence, machine learning, and robotics," Cutter Business Technology Journal, vol. 31, no. 2, pp. 4753, 2018.

[43] M. Nogel, G. Kovács, and G. Wersényi, "Regulation of Digital Reality in Nutshell," in 12th IEEE International Conference on Cognitive Infocommunications (CogInfoCom), pp. 1-7, IEEE, 2021.

[44] H.-K. Wu, S. W.-Y. Lee, H.-Y. Chang, and J.-C. Liang, "Current status, opportunities and challenges of augmented reality in education," Computers \& education, vol. 62, pp. 41-49, 2013.

[45] G. Makransky, S. Borre-Gude, and R. E. Mayer, "Motivational and cognitive benefits of training in immersive virtual reality based on multiple assessments," Journal of Computer Assisted Learning, vol. 35, no. 6, pp. 691-707, 2019.

[46] L. Freina and M. Ott, "A literature review on immersive virtual reality in education: state of the art and perspectives," The international scientific conference elearning and software for education, vol. 1, no. 133, pp. 10-1007, 2015.

[47] M. Handosa, H. Schulze, D. Gracanin, M. Tucker, and M. Manuel, “An Approach to Embodiment and Interactions with Digital Entities in Mixed-Reality Environments," in 2018 IEEE Conference on Virtual Reality and $3 D$ User Interfaces (VR), pp. 569-570, IEEE, 2018.

[48] Á. Kovács, "Evaluation of the Aggregation Capability of the MPT Network Layer Multipath Communication Library and Multipath TCP," Acta Polytechnica Hungarica, vol. 16, no. 6, pp. 129-147, 2019. 